\title{
Assessment of Water Quality of Yağlidere Stream (Turkey) Using Multivariate Statistical Techniquess
}

\author{
Arzu Aydin Uncumusaoğlu ${ }^{1 *}$, Tamer Akkan² \\ ${ }^{1}$ Department of Environmental Engineering, Faculty of Engineering, \\ Giresun University 28200, Giresun, Turkey \\ ${ }^{2}$ Department of Biology, Faculty of Arts and Sciences, \\ Giresun University, 28200, Giresun, Turkey
}

Received: 17 November 2016

Accepted: 13 February 2017

\begin{abstract}
Yağlıdere Stream is one of the major waterways flowing into the eastern Black Sea. In this study, multivariate statistical techniques, hierarchical cluster analysis (HCA), and principal component analysis (PCA) were applied to data on Yağlidere water quality. Thus, we aimed to determine main pollution factors and time risky polluted areas. During the study, water samples were taken by monitoring 23 physicochemical parameters at five different sites every month between June 2013 and May 2014. In addition, Pearson correlation was used to determine the relationships of all physico-chemical parameters. According to the results of HCA, five sampling areas were grouped into two clusters. From the PCA results, it may be estimated that river pollution is mainly from agricultural runoff and soil weathering, soil erosion, hydroelectric power plant installation activities, domestic disposal, and leaching from solid waste disposal sites. Consequently, the Yağlıdere is of good quality according to the physico-chemical data by national and international permissible limits, but it is under pressure. These temporal and spatial scale effects indicate that water-monitoring schemes need to be scaled-sensitive to water management for coming years.
\end{abstract}

Keywords: water quality, fresh water, principal component analysis (PCA), hierarchical cluster analysis (HCA), Pearson's correlation

\section{Introduction}

The surface water quality in a region largely depends on the nature and extent of the industrial, agricultural, and other anthropogenic activities in the catchments. The quality of these resources is a very sensitive issue. Anthropogenic influences as well as natural processes degrade surface waters and impair their use for drinking, industry, agriculture, recreation, and other purposes

*e-mail: arzu.a.uncumusaoglu@gmail.com
[1-2]. Pollution of surface water bodies with toxic chemicals and excess nutrients, resulting from water runoff and groundwater discharges, has been an issue of worldwide environmental concern. Nutrients like phosphorus and nitrogen support the growth of algae and other plants. However, excessive levels of nutrients from sources such as fertilizer can cause eutrophication in an aquatic ecosystem.

In particular, it is necessary to ensure continuous monitoring of water for good water management and because fresh water will be a scarce source in the future. Monitoring the quality of surface water will help protect 
our waterways from pollution. In order to understand the process dynamics of a watershed, a well-designed water quality monitoring network identifies water quality problems while establishing baseline values for short- and long-term trend analysis. Generally, fresh water quality is affected by excess domestic and solid waste. And it even occurs when pollutants (particles, chemicals, or substances that contaminate water) are discharged directly or indirectly into water bodies without enough treatment to get rid of harmful compounds. Pollutants get into water mainly by human causes or factors. Water pollution is usually caused by human activities. Different human sources add to the pollution of water.

In addition to monitoring research in recent years, principal component analysis (PCA) and hierarchical cluster analysis (HCA) have been widely used as they help with the interpretation of monitoring a network's complex data arrays to gain a better understanding of water quality. This analyzes river hydrochemistry problems, and helps to evaluate chemical species and sources of pollution associated with hydrologic conditions [2-8]. Also, these techniques allow for the identification of possible sources that affect water systems and provide valuable tools for the safe management of water resources and the analysis of data from non-homogeneous variables [9-11]. And they are useful in verifying temporal and spatial variations caused by natural and anthropogenic factors and PCA and HCA groups, and sets of variables with similar properties [11-12].

Giresun has various sources of drinking water, but it is undoubtedly of great importance to take measures to determine and protect the quality of these resources. However, with the rapid increase in the population of the city and the need to meet the increasing demands of irrigation and human consumption, the available water resources are getting depleted and water quality has deteriorated. In addition, the results of previous studies in this area also support our concerns about freshwater resources. The main source of livelihood for the people in question is agriculture and fishing. Especially Giresun hazelnut is an agricultural product known all over the world [13]. Chemical fertilizers and pesticides used unconsciously while hazelnut is grown are a great threat to water resources due to the frequent rainfall in the region. Since rivers constitute the main inland water sources for domestic and irrigation purposes, it is possible to ensure that river pollution is under control and that necessary precautions have been taken in advance in case of threats, but only by obtaining reliable information on water quality and pollution sources.

In this study, Yağlıdere water quality was primarily evaluated by a monitoring program. PCA and HCA multivariate techniques were applied to summarize the statistical correlation between the components in the water samples and to assess information about the similarity and dissimilarities among stations, to identify water quality variables for spatial differences, and also further to make sure of the impact of pollution sources on water quality parameters. It is also aimed at clarifying the impact of pollution sources on water quality parameters.

\section{Material and Methods}

\section{Sample Location and Sampling}

Yağlıdere Stream is formed by small streams joining together after originating from the Çakrak Mountains in the southeast border of Giresun Province, and the length of its main branch is $70 \mathrm{~km}$. After it is formed, the Yağlidere passes through towns and cities and pours into the southeastern Black Sea in the town of Espiye. This study

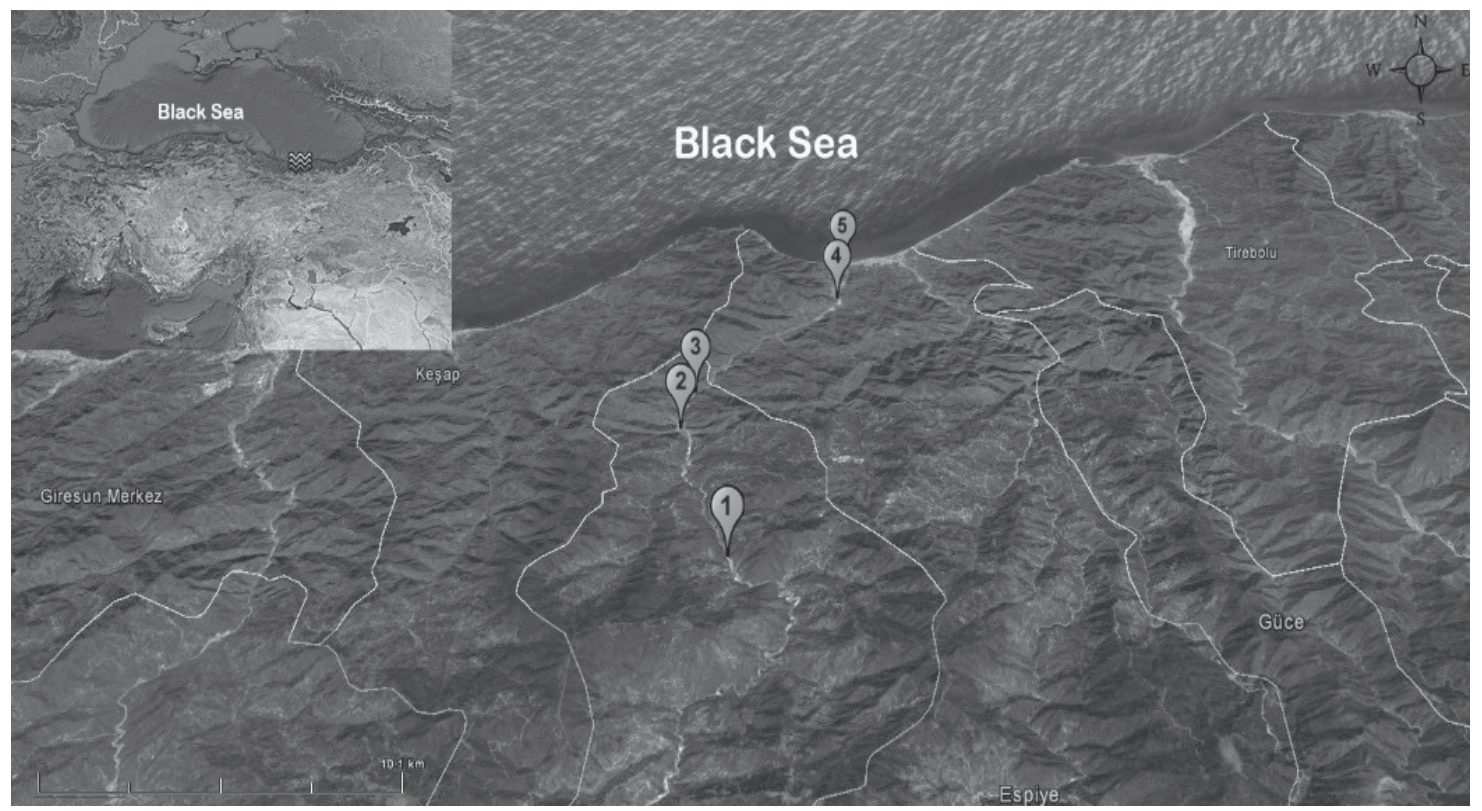

Fig. 1. Map of study area with sampling point locations (adapted from Google Earth). 
was conducted along the Yağlıdere in the city of Giresun. Replicate samples for each location along the stream were collected monthly in June 2013 and May 2014 from five stations (Fig. 1).

Samples for water quality studies were collected in $2.5 \mathrm{~L}$ plastic bottles that had been previously soaked in $5 \%$ nitric acid for $24 \mathrm{~h}$ and rinsed with distilled water. Moreover, water samples were collected in 1-liter pre cleaned (with $50 \% \mathrm{HNO}_{3}$ and then thrice with deionized water) polyethylene bottles and acidified with $10 \mathrm{~mL}$ concentrated $\mathrm{HNO}_{3}$ per liter of wastewater for the analysis of heavy metals. After collection, the samples were placed in coolers with ice bags while being transported to the laboratory and kept at about $4^{\circ} \mathrm{C}$ until analysis.

\section{Determination of Physico-Chemical Parameters}

Water temperature, conductivity, dissolved oxygen, $\mathrm{pH}$, total dissolved solid, salinity, and oxidoreduction potential were determined using a YSI 556 MPS meter, and turbidity was measured with Turb 355 T/IR turbid meter (WTW) after calibration with standard buffer solutions.

Routine laboratory analysis, such as total ammonia nitrogen, nitrite nitrogen, nitrate nitrogen, ammonium, ammonia, silica, total phosphorus, orthophosphate phosphorus, alkalinity, hardness, suspended solids, chlorophyll a and $\mathrm{BOD}_{5}$ were measured by standard methods [14]. To assess the precision and accuracy of results, replicate analysis of blank, standard, and samples were done.

\section{Statistical Analysis}

Statistical analysis of data was carried out using SPSS statistical package programs. Descriptive statistical analysis, including one-way ANOVA and significance (0.01 and 0.05) was done. Important differences in the mean values were tested with Tukey's multiple range test. Moreover, relationships among the considered variables were tested using Pearson's correlation. Multivariate analysis of the stream water quality data set was performed through principal component and hierarchical cluster analysis (PCA-HCA) techniques [23]. The water quality data had been initially standardized by z-scale transformation because the water quality variables were measured in different units [15-16]. All statistical calculations were performed with SPSS 17.0 for Windows.

\section{Result and Discussion}

The annual mean values of physico-chemical parameters ranged between, for WT: 7.02 and $24.41^{\circ} \mathrm{C}$, EC: 175 and $428 \mathrm{mS} / \mathrm{cm}$, TDS: 0.11 and $0.28 \mathrm{~g} / \mathrm{L}, \mathrm{pH}$ : 6.96 and 8.57 , DO: 7.04 and $15.52 \mathrm{mg} / \mathrm{L}$, salinity: 0.01 and $0.21 \mathrm{ppt}$, ORP: -77 and $133.20 \mathrm{mV}$, TAN: 0.106 and $5.577 \mathrm{mg} / \mathrm{L}, \mathrm{NO}_{3}-\mathrm{N}: 0.311$ and $2.100 \mathrm{mg} / \mathrm{L}, \mathrm{NO}_{2}-\mathrm{N}: 0.001$ and $0.038 \mathrm{mg} / \mathrm{L}, \mathrm{NH}_{3}: 0.001$ and $0.081 \mathrm{mg} / \mathrm{L}, \mathrm{NH}_{4}^{+}: 0.102$ and $5.542 \mathrm{mg} / \mathrm{L}$, silica: 6.6 and $18 \mathrm{mg} / \mathrm{L}, \mathrm{TP}: 0.011$ and $0.915 \mathrm{mg} / \mathrm{L}, \mathrm{O}-\mathrm{PO}_{4}: 0.008$ and $0.354 \mathrm{mg} / \mathrm{L}, \mathrm{Na}: 1.30$ and $9.70 \mathrm{mg} / \mathrm{L}, \mathrm{K}: 0.30$ and $10 \mathrm{mg} / \mathrm{L}$, alkalinity: 56 and $174 \mathrm{mg} / \mathrm{L}$, hardness: 9 and $23.70^{\circ} \mathrm{F}$, Chl-a: 0.019 and $5.558 \mu \mathrm{g} / \mathrm{L}$, turbidity: 1.920 and $74.540 \mathrm{NTU}$, TSS: 0.264 and $2 \mathrm{~g} / \mathrm{L}$ and $\mathrm{BOD}_{5}: 0.51$ and $11.72 \mathrm{mg} / \mathrm{L}$.

The lowest and highest values of physico-chemical parameters were determined as a station, respectively: TDS, ORP, K, alkalinity, hardness, TSS and EC, DO, salinity, TAN, $\mathrm{NO}_{2}, \mathrm{NH}_{4}$, silica, TP, O- $\mathrm{PO}_{4}$, alkalinity, hardness at Station 1; WT, EC, TDS, $\mathrm{NO}_{3}, \mathrm{NH}_{4}$, and $\mathrm{pH}$, ORP, Chl-a at Station 2; DO, TAN, Chl-a and $\mathrm{NO}_{3}$, $\mathrm{NH}_{3}, \mathrm{BOD}_{5}$ at Station 3; salinity, TP, $\mathrm{Na}$ and WT, $\mathrm{NO}_{2}$, $\mathrm{Na}$, TSS at Station 4; $\mathrm{pH}$, TAN, silica, O- $\mathrm{PO}_{4}$, turbidity, $\mathrm{BOD}_{5}$, and $\mathrm{K}$, turbidity at Station 5 . In addition, the mean values of physiochemical parameters at different stations are demonstrated in Table 1. Moreover, the results indicate that some water quality parameters in Yağlıdere surface water are suitable for similar previous studies in Table 2 [28-32].

According to Turkish legislation, it is very important to define and elaborate upon the quality of water before use for various purposes in light of physico-chemical parameters [17-18]. Water quality of surface water is classified into four groups. Yağlidere surface water is in good condition in terms of WT and $\mathrm{NO}_{3}-\mathrm{N}$ values according to RSWQM. All parameters based on average values were found to be Class I water quality except for $\mathrm{NO}_{2}-\mathrm{N}, \mathrm{TP}$, and $\mathrm{BOD}_{5}$. Nitrate nitrogen concentrations did not exceed water standards ( $>5 \mathrm{ppm}$ ) [17-19] and were generally well below this level, but sometimes TP and $\mathrm{NH}_{4}$ were Class IV. The use of agricultural fertilizers and urban sewage are believed to increase the ammonia nitrogen, nitrate, phosphate, and nitrite concentrations because the absence of freshwater plants might affect the increase in nitrogen ion concentrations in the stream. Moreover, a comparison of our data with the WHO [20] acceptable limits showed that our values for turbidity are higher than, and hardness, $\mathrm{pH}$, sodium, and TDS lower than international guidelines.

We conducted statistical analyses of 60 samples taken monthly from five stations. For the Anova analyses seasonal mean levels at each of the stations except TP were significantly different $(\mathrm{P}<0.05)$, but between the stations there were no significant differences throughout the sampling period $(\mathrm{P}>0.05)$ (Table 1).

The Pearson correlation analysis of the water quality parameters listed in Table 3 indicated the absence of a positive and good correlation (above 0.7), although there was less significant correlation between some parameters. The TDS showed significant and positive high correlation with EC $(r=0.965, \mathrm{P}>0.01)$. Salinity showed significant and positive high correlation with EC and TDS $(\mathrm{r}=0.970, \mathrm{r}=0.936, \mathrm{P}>0.01)$, which are responsible for water mineralization. $\mathrm{pH}$ showed significant and positive correlation with WT $(\mathrm{r}=0.857, \mathrm{P}>0.01)$. $\mathrm{NH}_{3}$ showed significant and positive correlation with $\mathrm{pH}$ $(\mathrm{r}=0.742, \mathrm{P}>0.01) . \mathrm{NH}_{4}$ showed significant and positive max correlation with TAN $(\mathrm{r}=1.000, \mathrm{P}>0.01)$. $\mathrm{NO}_{3}$ was 


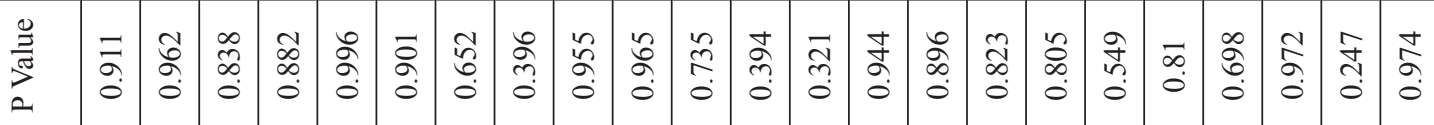

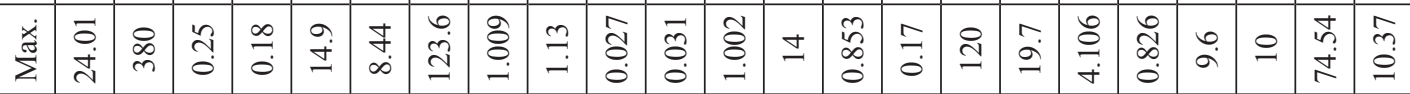

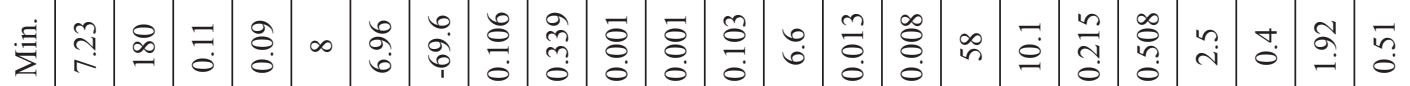

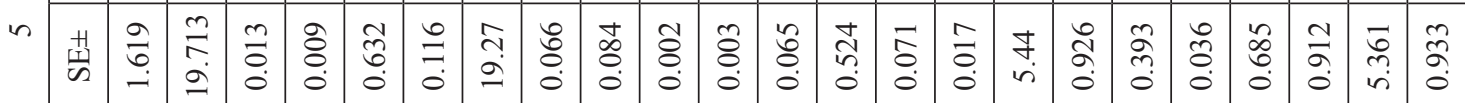

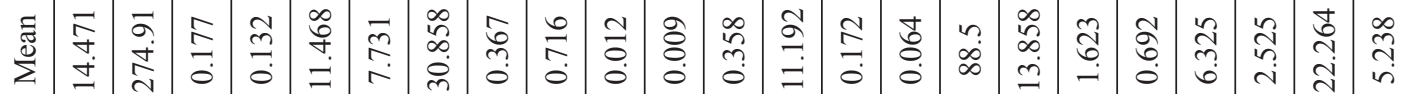

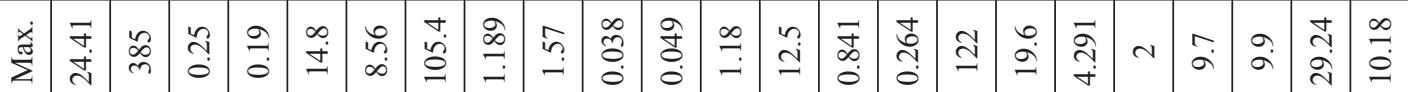

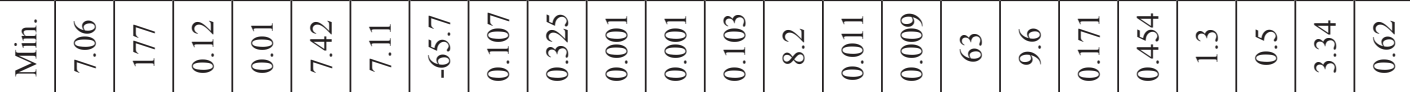

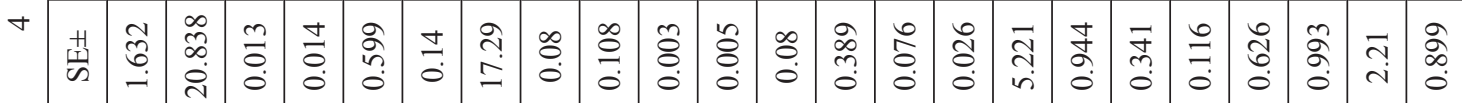

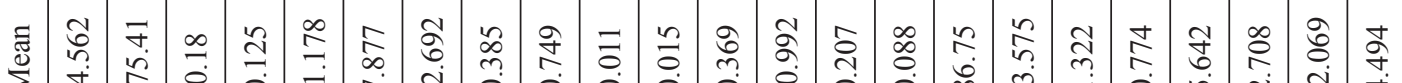

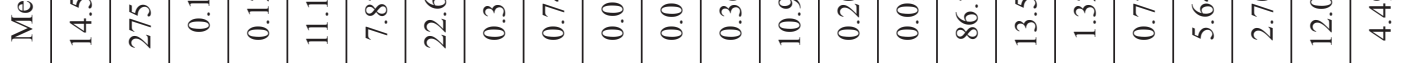

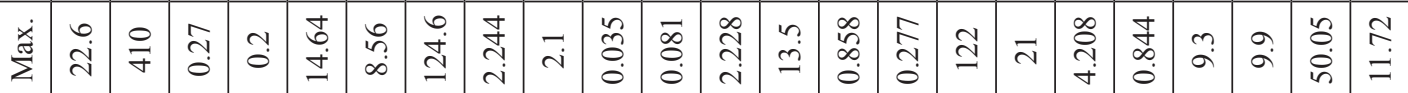

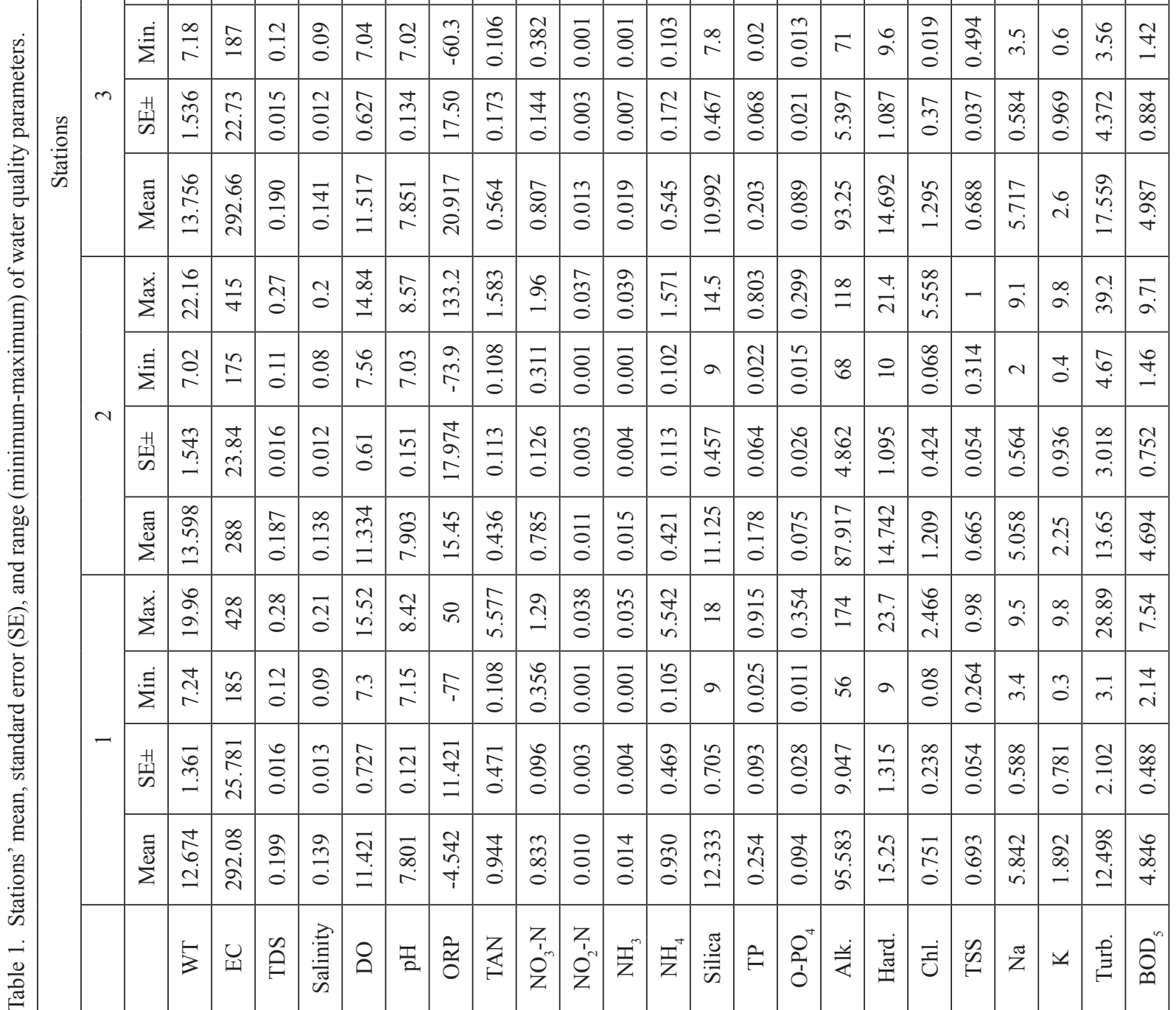


Table 2. Comparison of water quality parameters in previous studies.

\begin{tabular}{|c|c|c|c|c|c|}
\hline & $\begin{array}{c}\text { Gökpinar and } \\
\text { Çürüksu Creek [28] }\end{array}$ & $\begin{array}{c}\text { Kürk Stream } \\
{[29]}\end{array}$ & $\begin{array}{c}\text { Beydilli River } \\
{[30]}\end{array}$ & $\begin{array}{c}\text { Horohon Creek } \\
{[31]}\end{array}$ & $\begin{array}{l}\text { This } \\
\text { Study }\end{array}$ \\
\hline WT $\left({ }^{\circ} \mathrm{C}\right)$ & $10-28.4$ & $3.5-25.5$ & $3.8-26.4$ & $6-16$ & $7.02-24.41$ \\
\hline $\mathrm{pH}$ & $6.50-11.15$ & $7.1-8.9$ & $7.26-7.82$ & $8.11-8.47$ & $6.96-8.57$ \\
\hline DO (mg/L) & $4.5-8.2$ & $6.8-10.1$ & $10.29-14.90$ & $7.84-13.90$ & $7.04-15.52$ \\
\hline Salinity (ppt) & & & $0.01-0.08$ & $0.01-0.19$ & $0.01-0.21$ \\
\hline TDS (mg/L) & $295-3286$ & & & & $110-280$ \\
\hline $\mathrm{TSS}(\mathrm{mg} / \mathrm{L})$ & & & $0.1-4.9$ & $0.03-4.12$ & $264-2000$ \\
\hline Hardness $(\mathrm{mg} / \mathrm{L})$ & & $9.4-51$ & $99.42-155.26$ & & $9-23.7$ \\
\hline Alk. (mg/L) & & $89-689$ & & $137-250$ & $56-174$ \\
\hline $\mathrm{Na}(\mathrm{mg} / \mathrm{L})$ & & & $12.48-46.12$ & $7-77$ & $1.3-9.7$ \\
\hline $\mathrm{K}(\mathrm{mg} / \mathrm{L})$ & & & $1.27-3.06$ & $2-5.3$ & $0.3-10$ \\
\hline Turb. (NTU) & & $1-339$ & & & $1.92-74.54$ \\
\hline $\mathrm{NH}_{4}(\mathrm{mg} / \mathrm{L})$ & & & Nd-0.0013 & Nd-0.025 & $0.102-5.542$ \\
\hline $\mathrm{NO}_{2}-\mathrm{N}(\mathrm{mg} / \mathrm{L})$ & & & Nd-0.013 & Nd-0.009 & $0.001-0.038$ \\
\hline $\mathrm{NO}_{3}-\mathrm{N}(\mathrm{mg} / \mathrm{L})$ & & & Nd-0.95 & Nd-4.20 & $0.311-2.100$ \\
\hline $\mathrm{O}-\mathrm{PO}_{4}(\mathrm{mg} / \mathrm{L})$ & & & & & $0.008-0.354$ \\
\hline $\mathrm{BOD}_{5}(\mathrm{mg} / \mathrm{L})$ & $0.53-166$ & & $0.1-7.1$ & & $0.51-11.72$ \\
\hline
\end{tabular}

positively correlated with all parameters except $\mathrm{Na}$ and turbidity, which was a negative correlation - indicating that these variables are derived from similar sources and also moving together. The alkalinity showed significant and positive correlation with EC, TDS, and salinity $(r=0.829$, $\mathrm{r}=0.780, \mathrm{r}=0.795, \mathrm{P}>0.01) . \mathrm{NH}_{4}$ showed significant and positive max correlation with TAN $(\mathrm{r}=1.000, \mathrm{P}>0.01)$. Hardness showed significant and positive correlation with alkalinity $(\mathrm{r}=08.10, \mathrm{P}>0.01)$ and high positive correlation with EC, TDS, and salinity $(r=0.940, r=0.902$, $\mathrm{r}=0.901, \mathrm{P}>0.01)$. BOD showed significant and positive correlation with WT $(\mathrm{r}=0.718, \mathrm{P}>0.01)$. As expected, the negative relationship of DO with other parameters reveals the organic pollution that can be considered one of the causes of water ecological changes in the environment (Table 3).

Due to the complexity of the relationship between numerous water quality parameters, confidential information was extracted using principal component analysis (PCA) and revealed detailed data structure. PCA analysis was combined to help eliminate the redundant data and choose responsible environmental factors [21-22]. PCAs classified the factor loadings as 'strong,' 'moderate,' and 'weak,' corresponding to absolute loading values of $>0.75,0.75-0.50$, and $0.50-0.30$, respectively [23]. The results of calculations are shown in Table 4.

A PCA analysis included seven components, and 23 physico-chemical parameters were classified. These components were acquired with eigenvalues $>1$ summing $83.53 \%$ of the total variance in the surface water dataset (Table 4 and Figs 2-3, respectively). The first
PC, explaining $33.62 \%$ of the total variance, has a strong positive loading on EC, hardness, TDS, salinity, alkalinity, and moderate positive loadings on $\mathrm{BOD}_{5}$ and WT. This natural source of these parameters in catchments from soil weathering and flowing run-off. This factor can be based on seasonal changes [24].

The second PC, accounting for $14.86 \%$ of the total variance, has a strong positive loading on $\mathrm{pH}$. $\mathrm{NH}_{3}$ has strong negative loadings on dissolved oxygen and moderate positive loadings on WT. This factor represents the contribution of non-point source pollution from hazelnut trees. In these areas, farmers use nitrogenous fertilizer, which undergoes nitrification processes and the rivers receive nitrate nitrogen by groundwater leaching [4]. In addition, this factor represents loadings on a moderate negative temperature and dissolved oxygen [25-26].

The third PC, accounting for $10.28 \%$ of the total variance, has a strong positive loading on TAN and $\mathrm{NH}_{4}$, and moderate positive loadings on $\mathrm{K}$. This shows the influence of agricultural runoff from the soil as nitrogenous fertilizers are extensively used in this region - indicating the influence of domestic waste.

The fourth PC was explaining the lowest variance $(8.18 \%)$, which has strong positive loadings on $\mathrm{NO}_{3}-\mathrm{N}$ and moderate positive loadings on $\mathrm{NO}_{2}-\mathrm{N}$, and weak negative loadings on $\mathrm{Na}$. Thus it shows the nutrient group of pollutants, which points to some source of wastewater and runoff. This organic factor can be interpreted as representing influences from point sources, such as discharges from domestic wastewater and industrial effluents. 


\begin{tabular}{|c|c|c|c|c|c|c|c|c|c|c|c|c|c|c|c|c|c|c|c|c|c|c|c|c|}
\hline ిิ & & & & & & & & & & & & & & & & & & & & & & & & - \\
\hline 音 & & & & & & & & & & & & & & & & & & & & & & & - & $\begin{array}{l}\overrightarrow{0} \\
0 \\
\end{array}$ \\
\hline 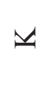 & & & & & & & & & & & & & & & & & & & & & & - & $\begin{array}{l}\frac{d}{0} \\
\frac{0}{0}\end{array}$ & \begin{tabular}{l} 
*a \\
\multicolumn{1}{c}{} \\
0 \\
$i$
\end{tabular} \\
\hline$\tilde{z}$ & & & & & & & & & & & & & & & & & & & & & - & $\begin{array}{l}n \\
0 \\
0 \\
1\end{array}$ & $\begin{array}{l}\infty \\
\stackrel{0}{0} \\
0\end{array}$ & $\begin{array}{l}\frac{*}{n} \\
\text { ma } \\
0\end{array}$ \\
\hline 婂 & & & & & & & & & & & & & & & & & & & & - & $\begin{array}{l}\tilde{0} \\
0 \\
0\end{array}$ & $\overrightarrow{0}$ & $\begin{array}{c}\tilde{0} \\
\tilde{0}\end{array}$ & 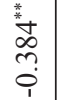 \\
\hline छ் & & & & & & & & & & & & & & & & & & & - & $\begin{array}{l}0 \\
\stackrel{0}{0} \\
0 \\
1\end{array}$ & 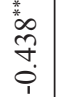 & $\begin{array}{l}0 \\
\stackrel{0}{0} \\
0\end{array}$ & त̂ & $\begin{array}{l}{ }_{\infty}^{*} \\
\infty \\
\infty \\
0 \\
0\end{array}$ \\
\hline 缡 & & & & & & & & & & & & & & & & & & - & 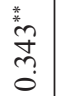 & 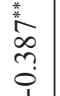 & 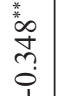 & $\begin{array}{l}\text { fo } \\
\dot{0} \\
\dot{\varphi}\end{array}$ & $\begin{array}{l}\infty \\
\infty \\
0 \\
1\end{array}$ & \begin{tabular}{l}
$*$ \\
\multirow{\sigma}{*}{}
\end{tabular} \\
\hline$\ddot{\vec{z}}$ & & & & & & & & & & & & & & & & & - & 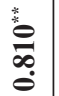 & 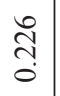 & 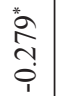 & \begin{tabular}{l}
0 \\
\multirow{2}{*}{} \\
\multirow{1}{1}{} \\
1
\end{tabular} & $\begin{array}{l}\hat{o} \\
\text { i. }\end{array}$ & $\frac{\beta}{0}$ & 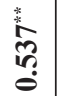 \\
\hline $\mathrm{O}^{+}$ & & & & & & & & & & & & & & & & - & $\begin{array}{l}m_{0}^{*} \\
\stackrel{f}{f} \\
0\end{array}$ & $\begin{array}{l}\text { : } \\
\stackrel{0}{0} \\
\stackrel{0}{0}\end{array}$ & $\begin{array}{c}\frac{*}{*} \\
\stackrel{+}{+} \\
0\end{array}$ & 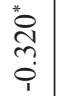 & 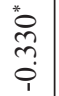 & $\begin{array}{l}\overrightarrow{0} \\
\dot{0}\end{array}$ & $\begin{array}{l}m \\
0 \\
0 \\
1\end{array}$ & 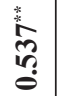 \\
\hline$\because$ & & & & & & & & & & & & & & & - & $\frac{\partial}{0}$ & $\begin{array}{l}8 \\
\stackrel{1}{0}\end{array}$ & $\begin{array}{l}\stackrel{?}{\mathrm{Z}} \\
\stackrel{0}{0}\end{array}$ & 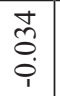 & $\begin{array}{l}\tilde{\hat{~}} \\
0\end{array}$ & $\begin{array}{l}0 \\
0 \\
0 \\
0 \\
1\end{array}$ & 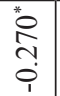 & $\begin{array}{l}\vec{n} \\
0 \\
i \\
1\end{array}$ & $\frac{\mathfrak{d}}{0}$ \\
\hline$\overline{\tilde{n}}$ & & & & & & & & & & & & & & - & $\begin{array}{l}\text { oे } \\
\text { iे }\end{array}$ & 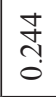 & $\begin{array}{l}\text { तิ } \\
\text { ○े }\end{array}$ & $\begin{array}{l}\stackrel{*}{*} \\
\infty \\
0 \\
0 \\
0\end{array}$ & $\begin{array}{l}\text { * } \\
\text { तิ } \\
0\end{array}$ & $\begin{array}{l}0 \\
0 \\
0 \\
0 \\
i\end{array}$ & $\begin{array}{l}\circ \\
0 \\
\stackrel{0}{1}\end{array}$ & $\begin{array}{l}\infty \\
\stackrel{0}{0} \\
0\end{array}$ & $\begin{array}{l}\tilde{2} \\
0 \\
\dot{1}\end{array}$ & : \\
\hline $\bar{z}^{+}$ & & & & & & & & & & & & & - & 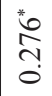 & $\begin{array}{l}\frac{t}{2} \\
\stackrel{+}{1}\end{array}$ & $\begin{array}{l}\vec{f} \\
\stackrel{0}{i}\end{array}$ & 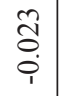 & \begin{tabular}{l}
$n$ \\
\multirow{2}{0}{} \\
0 \\
0
\end{tabular} & $\begin{array}{c}\vec{J} \\
\dot{i}\end{array}$ & $\begin{array}{l}\stackrel{\text { oे }}{0} \\
\dot{0}\end{array}$ & $\begin{array}{l}\tilde{n} \\
\stackrel{0}{0} \\
0\end{array}$ & 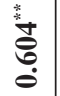 & $\begin{array}{l}n \\
\stackrel{n}{0} \\
0\end{array}$ & 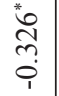 \\
\hline $\bar{z}^{n}$ & & & & & & & & & & & & - & तิ & 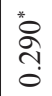 & ర్ & 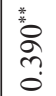 & 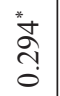 & \begin{tabular}{l}
$\stackrel{*}{\infty}$ \\
$\stackrel{\infty}{n ! n}$ \\
\hdashline
\end{tabular} & 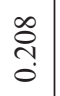 & $\begin{array}{l}{ }_{i}^{*} \\
\tilde{m}_{1} \\
0\end{array}$ & $\underset{0}{\overrightarrow{0}}$ & চ̋. & $\begin{array}{l}n \\
\text { ôे } \\
1\end{array}$ & $\begin{array}{l}\text { : } \\
\text { 省 } \\
0\end{array}$ \\
\hline$\stackrel{0}{z}^{\prime \prime}$ & & & & & & & & & & & - & $\stackrel{\circ}{\stackrel{2}{0}}$ & $\begin{array}{l}2 \\
\hat{\sigma} \\
0\end{array}$ & $\frac{2}{9}$ & $\begin{array}{l}\text { ते } \\
\text { त̂ }\end{array}$ & $\mid \begin{array}{l}* \\
0 \\
0 \\
0 \\
i\end{array}$ & $\begin{array}{c}m \\
\stackrel{1}{0}\end{array}$ & $\begin{array}{l}\stackrel{m}{\hat{n}} \\
\hat{\varphi}\end{array}$ & 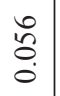 & $\begin{array}{l}\vec{\delta} \\
\stackrel{0}{i} \\
1\end{array}$ & 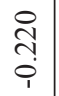 & 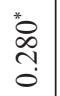 & $\begin{array}{l}\stackrel{\infty}{n} \\
\stackrel{0}{0}\end{array}$ & $\frac{\text { qu }}{\dot{q}}$ \\
\hline$\stackrel{0}{z}^{m}$ & & & & & & & & & & - & $\begin{array}{l}\text { 并 } \\
\stackrel{0}{0} \\
0\end{array}$ & $\begin{array}{l}\text { Iิ } \\
\stackrel{0}{0}\end{array}$ & $\begin{array}{l}\stackrel{\circ}{0} \\
\stackrel{0}{0}\end{array}$ & तั & $\stackrel{+}{\stackrel{t}{0}}$ & $\mid \begin{array}{l}\tilde{S} \\
0 \\
0\end{array}$ & $\begin{array}{l}\stackrel{+}{0} \\
\stackrel{0}{0}\end{array}$ & 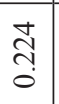 & \begin{tabular}{l} 
đ̊ \\
\multirow{2}{*}{} \\
0
\end{tabular} & 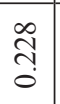 & 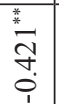 & 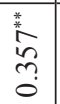 & $\begin{array}{l}0 \\
\stackrel{0}{1} \\
i \\
1\end{array}$ & $\stackrel{\infty}{\stackrel{\infty}{0}}$ \\
\hline 杂 & & & & & & & & & - & $\begin{array}{l}\mathbb{I} \\
\stackrel{Z}{0}\end{array}$ & $\frac{\overrightarrow{0}}{0}$ & 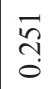 & : & $\begin{array}{l}\text { *) } \\
\stackrel{0}{0} \\
\stackrel{0}{0}\end{array}$ & $\frac{\widetilde{a}}{\stackrel{0}{1}}$ & $\begin{array}{l}m \\
\stackrel{0}{0} \\
\dot{p}\end{array}$ & $\begin{array}{l}\hat{a} \\
0 \\
\dot{0}\end{array}$ & $\begin{array}{l}n \\
\tilde{c} \\
0\end{array}$ & $\begin{array}{l}0 \\
\\
\stackrel{0}{1}\end{array}$ & $\begin{array}{l}\mathscr{2} \\
0 \\
0 \\
0\end{array}$ & $\begin{array}{l}+ \\
0 \\
0 \\
0\end{array}$ & \begin{tabular}{l}
\multirow{2}{*}{} \\
$\stackrel{0}{0}$ \\
$\stackrel{0}{0}$
\end{tabular} & $\begin{array}{l}\stackrel{0}{0} \\
0 \\
0\end{array}$ & 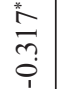 \\
\hline ๘े & & & & & & & - & & $\begin{array}{l}0 \\
\\
\\
\end{array}$ & \begin{tabular}{l}
$\infty$ \\
\multirow{1}{*}{} \\
0
\end{tabular} & $\begin{array}{l}\tilde{o} \\
\stackrel{i}{i}\end{array}$ & $\begin{array}{l}\frac{*}{*} \\
\frac{1}{n} \\
\vdots\end{array}$ & $\begin{array}{l}\vec{t} \\
\stackrel{0}{0}\end{array}$ & 辛 & $\begin{array}{l}\stackrel{\circ}{\circ} \stackrel{0}{i} \\
\end{array}$ & $\begin{array}{l}0 \\
\stackrel{0}{0} \\
0\end{array}$ & $\begin{array}{l}\text { 美 } \\
\text { oे } \\
\dot{0}\end{array}$ & 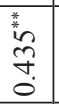 & $\begin{array}{l}\text { तิ } \\
\text { ○े }\end{array}$ & 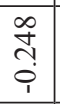 & 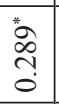 & ळे. & 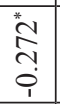 & $\begin{array}{l}0 \\
0 \\
0\end{array}$ \\
\hline 吉 & & & & & & - & ڤ్) & & $\begin{array}{l}\vec{a} \\
\dot{i} \\
\end{array}$ & $\begin{array}{l}\text { वे } \\
0 \\
0\end{array}$ & $\begin{array}{l}: \\
\stackrel{0}{0}\end{array}$ & $\stackrel{*}{\stackrel{*}{*}}$ & $\stackrel{\text { के }}{\vec{i}}$ & $\begin{array}{l}\hat{\infty} \\
\stackrel{0}{0}\end{array}$ & $\begin{array}{l}\text { तิ } \\
\text { ஸे }\end{array}$ & 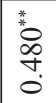 & $\begin{array}{l}\stackrel{8}{1} \\
0\end{array}$ & 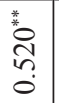 & $\begin{array}{l}\stackrel{*}{m} \\
\stackrel{7}{*} \\
0\end{array}$ & $\begin{array}{l}{ }^{*} \\
\text { के } \\
\text { fे }\end{array}$ & $\begin{array}{l}0 \\
\stackrel{1}{1} \\
i \\
1\end{array}$ & 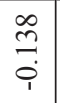 & $\begin{array}{l}\infty \\
\stackrel{0}{0} \\
\stackrel{i}{i}\end{array}$ & 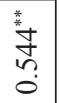 \\
\hline ๑ & & & & & - & $\begin{array}{l}\text { : } \\
\text { i. } \\
\text { i. }\end{array}$ & 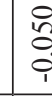 & & $\begin{array}{l}\hat{\text { ț }} \\
\text { है }\end{array}$ & $\begin{array}{l}\Delta \\
0 \\
0 \\
0\end{array}$ & 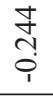 & 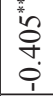 & $\begin{array}{l}\tilde{n} \\
\tilde{o}\end{array}$ & है & $\begin{array}{l}\vec{I} \\
\stackrel{i}{i}\end{array}$ & $\begin{array}{l}\frac{J}{9} \\
\end{array}$ & $\begin{array}{l} \pm \\
\vdots \\
0\end{array}$ & $\begin{array}{l}\text { an } \\
\stackrel{0}{9}\end{array}$ & 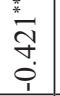 & \begin{tabular}{l} 
^̊ \\
\multirow{2}{0}{} \\
0
\end{tabular} & $\begin{array}{l}\infty \\
\stackrel{0}{0} \\
0\end{array}$ & $\frac{\mathfrak{g}}{0}$ & $\begin{array}{l}\text { ڤิ̀ } \\
\text { ọ }\end{array}$ & $\begin{array}{l}0 \\
\stackrel{0}{0} \\
\stackrel{0}{1}\end{array}$ \\
\hline ஜ் & & & & - & $\begin{array}{l}8 \\
\\
\end{array}$ & $\begin{array}{l}\text { in } \\
\text { in } \\
0\end{array}$ & $\begin{array}{l}* \\
\infty \\
0 \\
0\end{array}$ & & $\begin{array}{l}\infty \\
0 \\
0 \\
0\end{array}$ & $\begin{array}{l}0 \\
\stackrel{0}{0} \\
\vdots \\
0\end{array}$ & 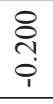 & : & $\begin{array}{l}\stackrel{\infty}{\stackrel{N}{*}} \\
\stackrel{0}{0}\end{array}$ & \begin{tabular}{l} 
尊 \\
\multirow{0}{*}{}
\end{tabular} & $\frac{\infty}{+}$ & 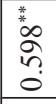 & 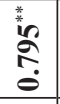 & 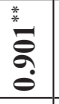 & 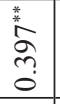 & 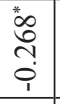 & 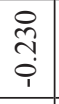 & $\stackrel{\hat{\sigma}}{0}$ & $\begin{array}{l} \pm \\
\vdots \\
i \\
\end{array}$ & 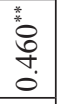 \\
\hline$\stackrel{\tilde{H}}{\Omega}$ & & & - & $\begin{array}{l}0 \\
0 \\
0\end{array}$ & 吕 & 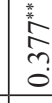 & $\mid \begin{array}{l}* \\
0 \\
0 \\
0 \\
0\end{array}$ & & $\frac{6}{6}$ & 离 & 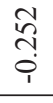 & $\begin{array}{l}0 \\
8 \\
8 \\
8\end{array}$ & $\stackrel{\overbrace{}}{\mathcal{I}}$ & 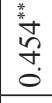 & $\stackrel{m}{=}$ & 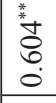 & $\begin{array}{l}\stackrel{0}{0} \\
\stackrel{0}{0} \\
\stackrel{0}{0}\end{array}$ & 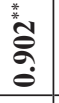 & $\begin{array}{l}\frac{*}{3} \\
\infty \\
\tilde{0} \\
0\end{array}$ & $\begin{array}{l}{ }^{\infty} \\
\text { ते } \\
\stackrel{1}{1}\end{array}$ & $\begin{array}{l}\text { ڤิ } \\
\stackrel{1}{1} \\
\stackrel{1}{1}\end{array}$ & $\begin{array}{l}\mathbb{1} \\
0 \\
0\end{array}$ & 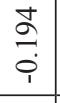 & 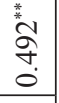 \\
\hline ن & & - & $\begin{array}{l}0 \\
6 \\
0 \\
0 \\
0\end{array}$ & : & 辇 & $\begin{array}{l}{ }_{0}^{*} \\
7 \\
0 \\
0\end{array}$ & $\mid \begin{array}{l}* \\
\vdots \\
\vdots \\
0\end{array}$ & & $\overrightarrow{\vec{v}}$ & 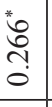 & $\begin{array}{l}\text { ते } \\
\text { î }\end{array}$ & 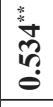 & 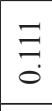 & $\begin{array}{l}\text { 䒺 } \\
\text { ô } \\
0 \\
0\end{array}$ & $\stackrel{\text { fo }}{\stackrel{0}{0}}$ & 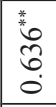 & 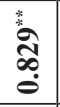 & $\begin{array}{l}0 \\
\stackrel{8}{0} \\
\stackrel{8}{8}\end{array}$ & 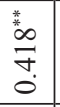 & $\begin{array}{l} \pm \\
\stackrel{1}{3} \\
\vdots \\
i\end{array}$ & \begin{tabular}{l}
$\infty$ \\
\multirow{1}{0}{} \\
$\vdots$ \\
$i$
\end{tabular} & oे & 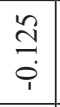 & $\begin{array}{l}3 \\
0 \\
0 \\
0\end{array}$ \\
\hline$\xi$ & - & $\begin{array}{l}\frac{\pi}{4} \\
\text { bे } \\
0\end{array}$ & 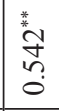 & $\begin{array}{l}\text { s. } \\
\text { in } \\
\text { ? }\end{array}$ & 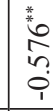 & 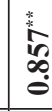 & $\frac{F^{*}}{\sigma}$ & & $\begin{array}{l}\hat{n} \\
\vdots \\
1 \\
1\end{array}$ & $\begin{array}{l}\tilde{2} \\
0 \\
0\end{array}$ & $\stackrel{n}{\circ}$ & : & 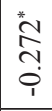 & 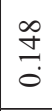 & $\frac{\tilde{\infty}}{0}$ & $\begin{array}{l}\text { t. } \\
\hat{n} \\
0 \\
0\end{array}$ & 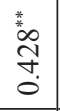 & $\begin{array}{l}x_{0}^{*} \\
0 \\
0 \\
0\end{array}$ & $\begin{array}{l}{ }^{*} 0 \\
\stackrel{n}{n} \\
0\end{array}$ & $\begin{array}{l}\stackrel{*}{*} \\
\stackrel{*}{+} \\
\stackrel{1}{+}\end{array}$ & 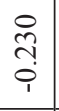 & 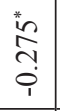 & $\begin{array}{l}\hat{m} \\
0 \\
0\end{array}$ & $\frac{*}{\stackrel{\infty}{*}}$ \\
\hline & 5 & U & $\tilde{\ominus}$ & तू & ๑ & t: & है & & Z & $\bigcap^{\prime}$ & $\stackrel{1}{\prime}^{\prime \prime}$ & $\overline{\mathbf{Z}}^{m}$ & $\bar{z}^{+}$ & $\dot{\bar{n}}$ & $\tilde{F}$ & $\stackrel{\circ}{+}^{+}$ & $\dot{\ddot{z}}$ & 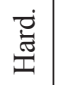 & छ் & $\begin{array}{l}\mathscr{W} \\
\tilde{\omega}\end{array}$ & $\tilde{z}$ & 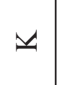 & 音 & Оి \\
\hline
\end{tabular}


Table 4. Varimax rotated factor matrix for the whole data set.

\begin{tabular}{|c|c|c|c|c|c|c|c|}
\hline Eigenvalues & 7.735 & 3.418 & 2.365 & 1.882 & 1.426 & 1.249 & 1.139 \\
\hline Percentage of variance & 33.629 & 14.860 & 10.284 & 8.181 & 6.200 & 5.429 & 4.950 \\
\hline \multirow{2}{*}{ Accumulative \% } & 33.629 & 48.489 & 58.773 & 66.954 & 73.154 & 78.583 & 83.533 \\
\hline & \multicolumn{7}{|c|}{ Factor loadings (varimax normalized) } \\
\hline $\mathrm{EC}$ & 0.94 & 0.183 & 0.086 & 0.077 & 0.178 & 0.051 & 0.019 \\
\hline Hardness & 0.931 & 0.264 & 0.024 & 0.034 & -0.023 & -0.059 & 0.043 \\
\hline TDS & 0.926 & 0.128 & 0.094 & 0.098 & 0.213 & -0.007 & 0.022 \\
\hline Salinity & 0.909 & 0.151 & 0.108 & 0.117 & 0.199 & 0.056 & 0.042 \\
\hline Alkalinity & 0.858 & -0.029 & -0.089 & 0.052 & 0.022 & -0.117 & -0.121 \\
\hline $\mathrm{O}-\mathrm{PO}_{4}$ & 0.705 & 0.283 & 0 & -0.161 & -0.32 & 0.125 & 0.117 \\
\hline BOD & 0.631 & 0.308 & -0.417 & -0.081 & -0.278 & -0.039 & -0.115 \\
\hline Silica & 0.435 & -0.027 & 0.288 & 0.012 & 0.41 & 0.349 & 0.185 \\
\hline $\mathrm{pH}$ & 0.297 & 0.908 & -0.089 & 0.05 & -0.079 & 0.024 & 0.015 \\
\hline DO & 0.08 & -0.783 & 0.186 & -0.106 & -0.014 & -0.314 & -0.048 \\
\hline $\mathrm{NH}_{3}$ & 0.364 & 0.757 & 0.27 & -0.009 & 0.282 & -0.132 & -0.041 \\
\hline WT & 0.52 & 0.744 & -0.283 & 0.012 & 0.026 & 0.071 & -0.053 \\
\hline TAN & 0.061 & -0.039 & 0.968 & -0.011 & -0.005 & -0.036 & -0.021 \\
\hline $\mathrm{NH}_{4}$ & 0.054 & -0.055 & 0.967 & -0.011 & -0.011 & -0.034 & -0.021 \\
\hline K & -0.039 & -0.134 & 0.711 & 0.384 & 0.095 & 0.166 & -0.142 \\
\hline $\mathrm{NO}_{3}-\mathrm{N}$ & 0.219 & -0.021 & 0.136 & 0.85 & 0.047 & -0.048 & 0.217 \\
\hline $\mathrm{NO}_{2}-\mathrm{N}$ & -0.359 & 0.316 & 0.139 & 0.655 & -0.088 & -0.047 & -0.365 \\
\hline ORP & 0.449 & 0.17 & -0.05 & 0.072 & 0.803 & -0.127 & -0.111 \\
\hline $\mathrm{Na}$ & -0.334 & -0.043 & 0.084 & -0.557 & 0.628 & -0.129 & -0.057 \\
\hline Turbidity & -0.197 & 0.065 & 0.113 & -0.166 & -0.143 & 0.764 & -0.191 \\
\hline Chlorophyll a & 0.374 & 0.283 & -0.192 & 0.331 & 0.012 & 0.649 & -0.002 \\
\hline $\mathrm{TP}$ & 0.128 & 0.204 & -0.184 & -0.03 & -0.106 & -0.255 & 0.803 \\
\hline TSS & -0.331 & -0.392 & 0.092 & 0.224 & 0.027 & 0.092 & 0.628 \\
\hline
\end{tabular}

Extraction Method: Principal Component Analysis. a. Rotation converged in 8 iterations.

Rotation Method: Varimax with Kaiser Normalization.
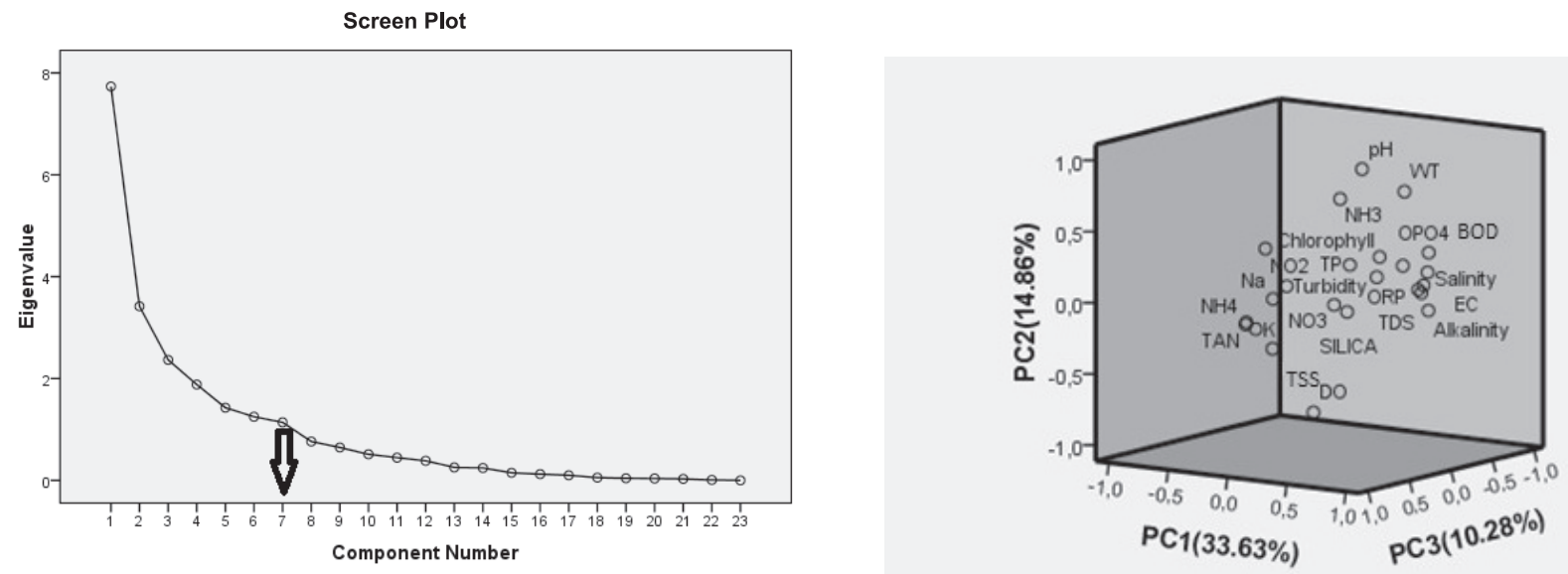

Fig. 2. Screen- plot for the principal component model of the monitoring data.

Fig. 3 Component plot. 


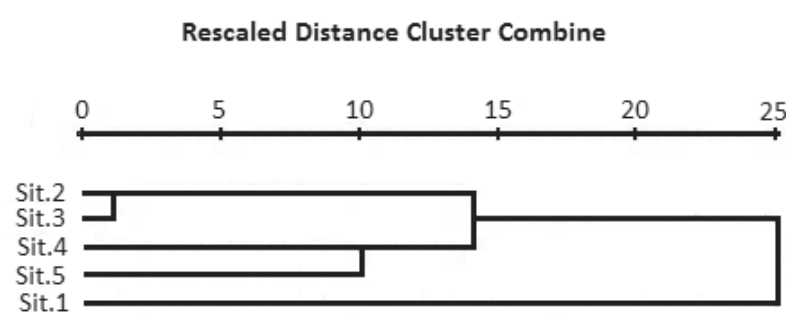

Fig. 4. Dendogram (using Ward Method) showing clusters of variables.

The fifth PC accounting for $6.20 \%$ of the total variance has a strong positive loading on ORP. This factor represents anthropogenic pollution sources and can explain the high levels of dissolved organic matter that consume large amounts of oxygen and that undergoe anaerobic fermentation processes leading to the formation of ammonia and organic acids.

The sixth PC accounts for $5.42 \%$ of the total variance and has a strong positive loading on turbidity and moderate positive loading on chlorophyll a due to the erosion and hydroelectric power plant installation activities in this region.

The seventh PC accounts for $4.95 \%$ of the total variance has a strong positive loading on TP and moderate positive loading on the suspended solid matter. The phosphate has its origin in soils due to use of phosphatic fertilizers in this region.

In this study, hierarchical cluster analysis (HCA) was used to show sampling stations with similar characteristics in the river basin. When HCA was applied for five stations and the dendogram (Fig. 4), hierarchical cluster analysis grouped five sampling sites into two clusters. The first cluster corresponds to station 1, situated at the most upstream site of the stream. This group makes understanding the self-purification and assimilative capacity of the stream because it almost does not receive pollution. This station is the nearest water source in order that it differs from other stations in terms of water quality.

Cluster 2 corresponds to (5 and 4, 2,3). These stations are situated at the most downstream sampling location in this river so receive pollution mostly from domestic wastewater. These stations receive pollution from nonpoint sources, but mostly from agricultural runoff and soil weathering, soil erosion, hydroelectric power plant installation activities, domestic disposal, and leaching from solid waste disposal sites. They have similar characteristics with wastes showing that these systems are highly affected by these pollution sources.

\section{Conclusion}

In this study the water quality level of Yağlidere Stream was analyzed and distributed pollution degrees using statistical analysis. Considering the increase of $\mathrm{NH}_{4}$ (at stations 1 and 3) and TP (at all stations), concentrations were evaluated by RSWQM as Class IV. Cluster analysis grouped five sampling sites into two clusters according to similar water quality characteristics.

From the PCA loadings it is evident that for this region major groups of parameter (strong loadings) that emerged are nutrient parameter group (representing influences from non-point sources such as atmospheric deposition and agricultural runoff) and organic pollution group (representing influences from point sources such as village and districts), and solid group (soil erosion and hydroelectric power plant installation activities by runoff process). PCA results were confirmed with HCA. It has emerged that these important pollutants in pollution sources have to be monitored regularly. From the PCA results, it may be persuasively estimated that the river pollution is mainly from agricultural runoff and soil weathering, soil erosion, hydroelectric power plant installation activities, domestic disposal, and leaching from solid waste disposal sites. In many countries an increasing proportion of water pollution originates from diffuse sources such as agricultural use of fertilizers, which cannot be controlled. Instead, the principle of "best environmental practice" should be applied to minimize non-point source pollution [27].

By this investigation we also suggest that precautions be taken in order to prevent future surface water pollution. Otherwise, such pollution can be dangerous for human health.

\section{Acknowledgements}

We would like to thank BAPKOM (Giresun University) for providing financial support for this project (No. FENBAP-A-220413-33).

\section{References}

1. CARPENTER S.R., COLE J.J., ESSINGTON T.E., HODGSON J.R., HOUSER J.N., KITCHELL J.F., PACE M.L. Evaluating alternative explanations in ecosystem experiments. Ecosystems, 1 (4), 335, 1998.

2. SIMEONOV V., STRATIS J.A., SAMARA C., ZACHARIADIS G., VOUTSA D., ANTHEMIDIS A., SOFONIOU M., KOUIMTZIS T. Assessment of the surface water quality in Northern Greece. Water Research, 37 (17), 4119-, 2003.

3. VEGA M., PARDA R., BARRADA E., DEBAN L. Assessment of seasonal and polluting effects on the quality of river water by exploratory data analysis. Water Research, 32, 3581, 1998.

4. SHRESTHA S., KAZAMA F. Assessment of surface water quality using multivariate statistical techniques: A case study of the Fuji River basin, Japan. Environmental Modelling and Software, 22 (4), 464, 2007.

5. FAN X., CUI B., ZHAO H., ZHANG Z., ZHANG H. School Assessment of river water quality in Pearl River Delta using multivariate statistical techniques. Procedia Environmental Sciences 2, 1220, 2010.

6. VIEIRA J.S., PIRES J.C., MARTINS F.G., VILAR V.J., BOAVENTURA R.A., BOTELHO C.M. Surface water 
quality assessment of Lis River using multivariate statistical methods. Water, Air, \& Soil Pollution, 223, 5549, 2012.

7. AZHAR S.C., ARISA A.Z., YUSOFFA M.K., RAMLIA M.F., JUAHIRC H. Classification of river water quality using multivariate analysis, Procedia Environmental Sciences 30, 79, 2015.

8. JUNG K.Y., LEE K.L., IMA T.H., LEE J.I., KIMA S., HANB K.Y., AHNA J. M. Evaluation of water quality for the Nakdong River watershed using multivariate analysis. Environmental Technology \& Innovation, 5, 67, 2016.

9. KAZI T.G., ARAIN M.B., JAMALI M.K., JALBANI N., AFRIDI H.I., SARFRAZ R.A., BAIG J.A., SHAH A.Q. Assessment of water quality of polluted lake using multivariate statistical techniques: A case study, Ecotox. Environ. Safe. 72, 301. 2009.

10. VIALLE C., SABLAYROLLES C., LOVERA M., JACOB S., HUAU M., MONTREJAUD-VIGNOLES M. Monitoring of water quality from roof runoff: Interpretation using multivariate analysis. Water Research, 45, 3765, 2011.

11. PALÁCIO S.M., ESPINOZA-QUIÑONES F.R.E., DE PAULI A.R., QUEIROZ C.B., FABRIS S.C., FAGUNDESKLEN M.R., VEIT M., PIANA P.A. Assessment of anthropogenic impacts on the water quality of Marreco River, Brazil, based on principal component analysis and toxicological assays. Water Air Soil Pollution, 227, 2016.

12. MUANGTHONG S., SHRESTHA S. Assessment of surface water quality using multivariate statistical techniques: case study of the Nampong River and Songkhram River, Thailand. Environmental Monitoring and Assessment, 187 (9), 548, 2015.

13. MALHOTRA S.P. World edible nuts economy, Concept publishing company, India. 533, 2008.

14. APHA. American water works association, water environment federation. Standard methods for the examination of water and wastewater. $19^{\text {th }}$ ed. Washington, 1995.

15. KANNEL P.R., LEE S., KANEL S.R., KHAN S.P. Chemometric application in classification and assessment of monitoring locations of an urban river system, Anal. Chim. Acta, 582, 390, 2007.

16. BU H.M., TAN X., LI S.Y., ZHANG Q.F. Water quality assessment of the Jinshui River (China) using multivariate statistical techniques, Environ. Earth Sci. 60, 1631, 2010.

17. RSWQM. Regulation on the surface water quality management. number of official gazette: 29327, Turkey, 2015.

18. ÖZDEMIR Ö. Application of multivariate statistical methods for water quality assessment of Karasu-Sarmisakli Creeks and Kizilirmak River in Kayseri, Turkey. Polish Journal of Environmental Studies, 25 (3), 1149, 2016.

19. KURNAZ A., MUTLU E., AYDIN UNCUMUSAOĞLU A. Determination of water quality parameters and heavy metal contet in surface water of Çiğdem Pond (Kastamonu/ Turkey). Turkish Journal of Agriculture-Food Science and Technology, 4 (10), 907, 2016.

20. WHO. Guidelines for Drinking-Water Quality, 4th Ed., Geneva, Switzerland, 2011.

21. SINGH K.P., MALIK A., MOHAN D., SINHA S. Multivariate statistical techniques for the evaluation of spatial and temporal variations in water quality of Gomti River (India) - a case study. Water Research, 38, (18), 3980, 2004.

22. OUYANG Y., NKEDI-KIZZA P., WU Q.T., SHINDE D., HUANG C. H. Assessment of seasonal variations in surface water quality. Water Research, 40 (20), 3800, 2006.

23. LIU C., LIN K., KUO Y. Application of factor analysis in the assessment of groundwater quality in a blackfoot disease area in Taiwan. Science of The Total Environment, 1, 313 (1-3), 77, 2003.

24. SINGH K.P., MALIK A., SINHA S. Water quality assessment and apportionment of pollution sources of Gomti River (India) using multivariate statistical techniques - a case study. Analytica Chimica Acta, 538, (1-2), 355, 2005.

25. LI B., PU P.M., HAN A.M. Factor analysis of water quality in Hongze Lake, China Environ. Sci., 1, 69, 2003.

26. THAREJA S., CHOUDHURY S., TRIVEDI P. Assessment of water quality of Ganga River in Kanpur by using principal components analysis. Advances in Applied Science Research, 2 (5), 84, 2011.

27. UNECE. Protection of water resources and aquatic ecosystems. Water Series No. 1. ECE/ENVWA/31. United Nations Economic Commission for Europe. New York. 1993.

28. TURAN F., ÜLKÜ G. Gökpinar ve Çürüksu çaylarinin kirlilik parametre ve yüklerinin izlenmesi. Pamukkale University Journal of Engineering Sciences, 19 (3), 133, 2013.

29. ŞEN B., GÖLBAŞI S. Hazar Gölü'ne dökülen Kürk Çayı'nın bazı fiziksel ve kimyasal özellikleri. E.U. Journal of Fisheries \& Aquatic Sciences, 25 (4), 353, 2008.

30. MUTLU E., KUTLU B., DEMIR T., YANIK T., SUTAN N.A. The evaluation of water quality parameters of Beydilli River (Hafik-Sivas). Journal of Selçuk University Natural and Applied Science, 40, 2014.

31. MUTLU E., YANIK T., DEMIR T. Horohon Deresi (HafikSivas) su kalitesi özelliklerinin aylık değişimleri. Alınteri Zirai Bilimleri Dergisi, 25 (B), 45, 2013.

32. VEREP B., AKIN S., MUTLU C., APAYDIN G., ERTUĞRAL B., ÇEVIK U. Assesment of trace elements in rainbow trout (Onchorhynchus mykiss) cultured in marine aquaculture cages on the Black Sea Coast. Fresenius Environmental Bulletin, 16 (9a), 1005, 2007. 